\title{
Towards a New Platform Based on Web 2.0 Technologies Supporting Collaborative Clinical Reasoning Behavior Skills
}

\author{
https://doi.org/10.3991/ijet.v16i08.19471 \\ Teldja Gherib $\left.{ }^{(}\right)$, Tahar Bouhadada \\ Badji Mokhtar - Annaba University, Annaba, Algeria \\ teldja.gheribeuniv-annaba.org
}

\begin{abstract}
Clinical Reasoning (CR) is a complex skill that must be learned by students during their training. However, it is difficult to learn and it is not immediately apparent how training should be organized to develop and improve students' clinical reasoning behavior. The use of Web 2.0 technologies in e-learning and e-health provides opportunities to consider effective teaching and learning methods, with an emphasis on collaboration. The importance of collaboration in medical education and practice is growing, and by working together professionals can build a better future. When a shared information need becomes a collaborative site, it is called Collaborative Information Behavior (CIB). To date, there is no system that fully supports CIB in clinical reasoning learning. This paper focuses on studies conducted in the field of CIB. In addition, research related to the clinical reasoning learning in medical pedagogy is also considered. A model dedicated to the design of a collaborative clinical reasoning behavioral environment in synchronous mode is proposed. The proposed model was concretized by a collaborative environment supporting behavioral competencies in collaborative clinical reasoning based on Web 2.0 technologies (MEDcollab), which aims to make student learning visible and accessible to educators.
\end{abstract}

Keywords - Clinical reasoning, collaborative environment, medical education, web 2.0 technologies, e-learning

\section{$1 \quad$ Introduction}

Clinical Reasoning (CR) is a complex skill that must be learned by medical students during their training [1]. It plays a significant role as it is the only link between medical knowledge and medical practice [2]. Although it is challenging learning it is not clear how training should be organized to develop and improve students' clinical reasoning behavior [3].

Clinical reasoning is known as the "thinking and decision-making processes associated with clinical practice" [4]. Carr defined it as "a cognitive process by which knowledge and experience are applied to clinical situations to develop solutions" [5]. Based on the two previous definitions, clinical reasoning is an abstract concept, and it is not explained. It is difficult for professionals to make this process explicit, and it is 
more difficult for medical students in training to understand the different forms of clinical reasoning processes of their tutors, and for medical educators to support students' clinical reasoning skills [6]. It is so abstract that the main barriers to medical education are its learning and evaluation [7].

Thus, medical students need to develop this crucial capacity and strive to strengthen it in the professional context [8]. The development of clinical reasoning skills for medical students is a key objective of medical education [9]. Clinical reasoning can be improved by motivating students to participate more actively in learning activities [10] [11] and [12].

Most medical universities have integrated teaching and learning activities such as clinical reasoning sessions (CRL). During CRL sessions, students simulate a medical consultation. It is difficult to find the ideal conditions to organize these sessions, although they have many advantages, and there are some limitations that limit the role of CRL meetings in clinical reasoning learning:

- The geographical distance between tutor and students

- Insufficient student experience and suboptimal supervision

- The evaluation of students by the same tutor at the CRL meeting is a negative point that can discourage the student from making mistakes, to the point of being inhibited in learning and not making the most of the learning situation [13]

- The limited number and type of patients available for treatment, decentralized observation, and opportunities for input and reflection [14].

E-learning is being used increasingly in higher education. [15]. Thus, the use of Web 2.0 technologies in CRL meetings provides an opportunity to consider new CCRL2.0 teaching and learning practices that can reinforce gaps in medical pedagogy. Various models of clinical reasoning have been developed so far, including a complex model called Modelling using Typed Objects (MOT); the model recognizes the complexity of the clinical reasoning process and the components that are involved [6]; an educational model for undergraduate psychiatry students, based on the use of real clinical cases and designed to develop and improve diagnostic clinical reasoning skills [16]; And an intervention model of clinical reasoning seminar intervention based on real cases; it aims is to study the importance of integrating CRL in initial medical training to give students an explicit overview of their reasoning [11].

The model for assessing behavioral skills in CR has not yet been identified in medical education and may be difficult to implement. Thus, this work aims to propose a model dedicated to the design of collaborative clinical reasoning behavior environment in synchronous mode and to concretize the model proposed by a collaborative environment "MEDcollab" supporting behavioral skills for collaborative clinical reasoning based on Web 2.0 technologies. To this end, the concepts of Collaborative Information Behavior (CIB) are combined with those of the CRL meeting.

In medical education, collaboration is becoming increasingly important [17]. Collaboration is a term commonly used in research, clinical practice, and health professions education. Through collaboration, we can work together for a better future [18]. When a shared information need becomes a collaborative site, it is called Collaborative Information Behavior (CIB) [19]. In this work, the Karunakaran's definition of CIB is 
adopted ("totality of behavior exhibited when people work together to identify an information need, retrieve, seek and share information, evaluate, synthesize and make sense of the found information, and then utilize the found information") [20]. Collaborative learning is a method involving a group of students working together to acquire skills or expert knowledge [21]. It is used extensively in learning institutions to promote knowledge transfer [22]. It would therefore be very useful to propose a model to promote collaborative CRL and to evaluate this capacity.

\section{The Proposed Collaborative Model for e-Learning to Improve Clinical Reasoning (CR) Behaviors for e-Health Environment}

This section describes the proposed collaborative model that supports the clinical reasoning learning as a collaborative activity between geographically distant clinicians (students, tutors) to help tutors better support the clinical reasoning of their trainees, and to help students structure, understand and interpret their thinking and approach. The model is based on the CIB research of Karunakaran. It also considers research involving the clinical reasoning learning in medical pedagogy. Moreover, the model takes into account collaborative research aimed at understanding the behavior of users who search for information in a collaborative environment, usually in digital environments [20]. This model is situated in the organizational context. However, their model needs to be tested and refined through more empirical examination in a variety of contexts.

In the proposed collaborative model strategy, important triggers work on a critical transition from individual to collaborative learning. These triggers push the individual to collaborate with others to achieve his/her goal quickly and effectively [20]. In addition, during each phase of the collaborative clinical reasoning behavior, students are guided and receive feedback from the tutor on their reasoning by providing meaningful and useful information [23]. The tutor thus encourages students to focus exclusively on the most relevant diagnosis. If they make a mistake, feedback from the tutor will direct specific knowledge needs, questioning, acknowledge and highlight not only the misdiagnosis but also the lack of information and help students to refine their learning needs. The tutor provides additional learning needs that students have not identified. They receive feedback from the tutor on their performance; the tutor invites them to verbalize explicitly and justify their intervention by giving an explanation of the different proposals, and encourages the students to focus exclusively on the most relevant hypothesis.

The model includes a set of activities that take place in three phases: the individual learning phase, the collaborative learning phase and the evaluation and synthesis phase. Some activities are specific to a particular phase, while others are common to all phases. The model helps to explain how these constituent activities relate to each other, and how collaboration is also a key element in making the clinical reasoning process explicit. Students have the opportunity to practice each phase collaboratively, validate their clinical competence through feedback from the collaborative tutor, discuss, search, 
synthesize and evaluate what they have learned, reorganize and activate their knowledge. Figure 1 illustrates how these sets of activities relate to each other.

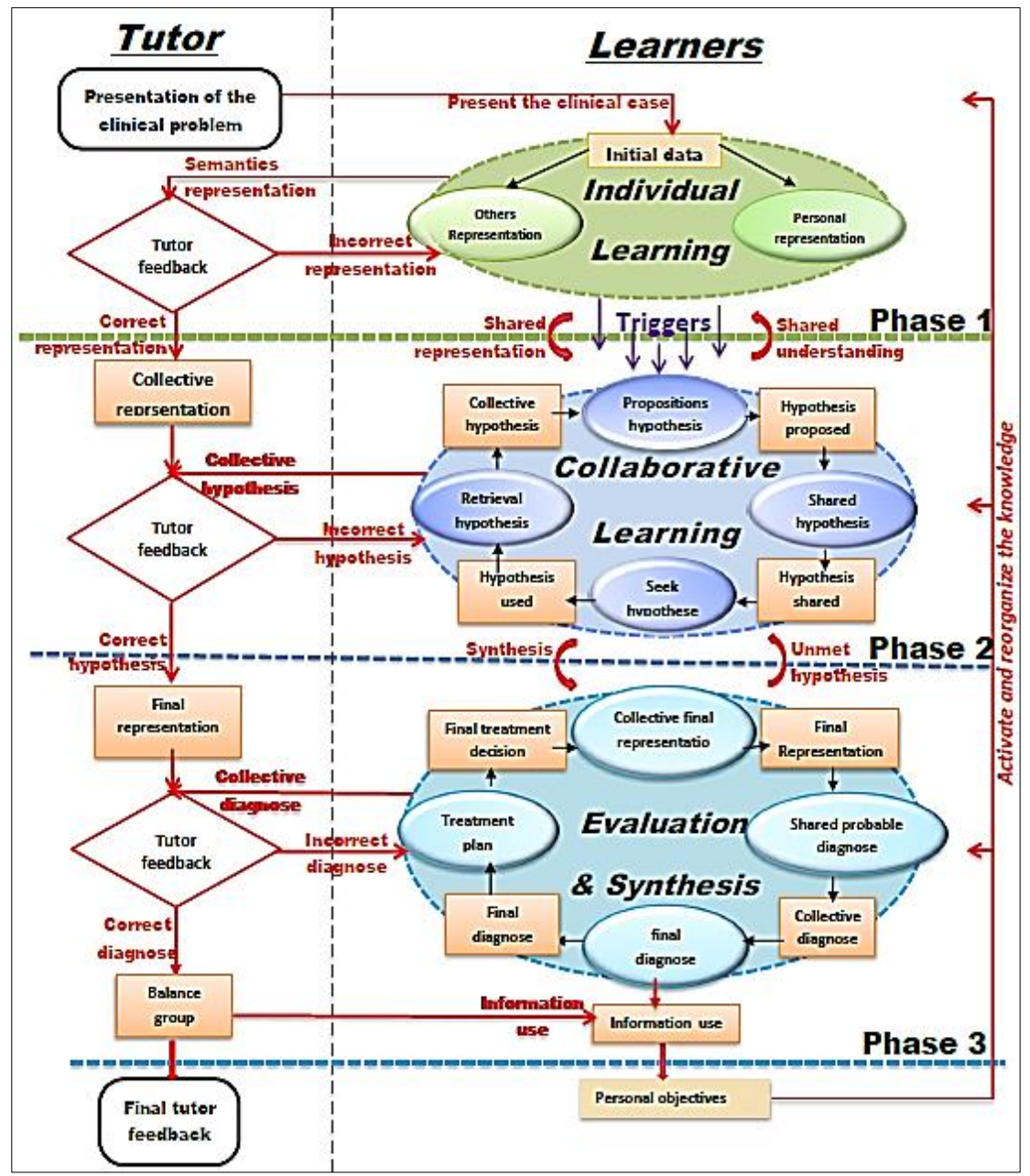

Fig. 1. Collaborative learning model to improve clinical reasoning behaviors skills for e-health environment

\subsection{Phase 1: Individual learning}

Phase 1 starts with the process of representing the problem. A clinical case problem will be addressed to students in the form of a patient report [24]. This is prepared by the tutor who will supervise each phase. Each clinical case contains sufficient information to allow students to establish a relevant diagnosis (patient name, age, sex, patient history, laboratory tests, etc.). The tutor acts as a source of clinical data from the patient. After the presentation of the clinical case by the tutor, each student builds his 
own semantic representation and creates a shared representation (initial representation). The individual learning phase is the first steps of the proposed collaborative model, where students consider their cognition and metacognition to define or represent the problem. The ideal time of learning is when the student connects new information to the prior knowledge network [25].

The sharing of semantic representations plays an important role as they serve as critical transition points between individual and collaborative learning to interact and discuss the shared representations to locate the most relevant ones. This transition occurs through a set of "triggers". These triggers include: knowledge gaps, lack of expertise, variance of shared semantic representations. Students cannot proceed with this phase until the specified individual activities (personal semantic representation) have been completed accurately. Feedback from the tutor on the student's semantic representations can be useful to share relevant semantic representations and trigger the next phase.

\subsection{Phase 2: Collaborative learning}

The second phase of collaborative learning is triggered when students who share their relevant semantic representation are validated by the tutor. In this case, several students collaborate and negotiate their shared representation to choose the most relevant one to establish a relevant diagnosis and improve their skills. The objective of the collaborative learning phase is to generate a common understanding of the clinical case problem. Moreover, collaborators typically create an explicit shared representation to achieve a shared understanding [26].

The performance of the next process is collaboratively analyzed and synthesized, and students are expected to generate hypotheses. They must propose a hypothesis, share different hypotheses, search for hypotheses, and retrieve relevant hypotheses. Through an iterative practice: research-sharing-evaluation-use-research until the relevant hypothesis is identified. This iterative strategy offers students the opportunity to collaborate and communicate their proposals with the goal of improving their approach to problem-solving Feedback from the tutor is the key reference for students to make their fundamental decisions during this phase, during which students must demonstrate their ability to make a relevant diagnosis.

This phase includes the following steps:

Proposition of hypothesis: Based on the case information and relevant knowledge, the student generates one or more hypotheses, and represents them as the proposed hypotheses, proposes a hypothesis to explain or solve the situation. All "relevant" and "irrelevant" hypotheses are stored in the system database. Students can collaborate with tutors or other students in groups to gain the necessary additional knowledge. The student works through the shared hypothesis and justifies or evaluates it by increasing the probability of a relevant hypothesis.

Share hypothesis: This step allows students to share their hypotheses and gather the opinions of their peers in the group. Students can share the proposed hypothesis with each other in a sharing space. They first choose the hypothesis they want to share from the proposed collective hypothesis, and then choose the hypothesis to share. The results (shared hypothesis) will appear in the sharing space for all students in the group 
Retrieval hypothesis: This step evaluates the shared hypothesis based on collaboration, additional data and tutor feedback, and develops further hypotheses if necessary, or uses the relevant shared hypothesis. The hypothesis used will appear in the space used by all students. The tutor encourages students to use only the most relevant hypothesis.

Final hypothesis: Students re-evaluate and synthesize the most relevant hypotheses. The tutor's comments are the key reference for the students to validate their final representation. The resulting collective final hypothesis is also compared and evaluated collaboratively for common understanding and use. Iterative clinical actions are required to explore the information required before a final representation is made.

Seek hypothesis: Students conduct a search in a common space through an iterative practice of searching-sharing-evaluation-used-searching until the necessary hypothesis is located.

\subsection{Phase 3: Synthesis and evaluation}

Students cannot proceed to this phase until the collective hypothesis has been adequately validated collaboratively. The results of the previous phase are evaluated and synthesized collaboratively, using the most relevant ones. They must formulate a final diagnosis. Students must propose a probable diagnosis, share different probable diagnoses, search for a probable diagnosis, evaluate a shared probable diagnosis, and retrieve the relevant diagnosis to specify a final treatment decision. An iterative practice of the students searching-sharing-evaluation-used-searching is used until the final diagnosis is located.

Thus, this iterative strategy is a fundamental factor in appropriate diagnosis. All probable "relevant" and "irrelevant" diagnoses are stored. In addition, students must decide what information they need to obtain to improve and develop the various diagnoses by increasing the likelihood of a relevant diagnosis. Students use the clues obtained from these data to synthesize and decide on their final diagnosis. The tutor asks the students to synthesize the problem and discuss the diagnostic path adopted by the group to develop a final collective diagnosis.

This phase includes the following steps:

Proposition diagnosis: Students are expected to establish a strong medical diagnosis. All proposed "relevant" and "irrelevant" diagnoses are stored in the system's database. Students can collaborate with the tutor or other students to obtain any additional information required.

Share diagnosis: Students can share the proposed diagnosis among themselves in the sharing space. They select the probable diagnosis if they want to share it from the proposed collective diagnosis and then choose "Share Diagnosis". The results will appear in the sharing space for all the students in the group.

Seek diagnosis: Students search in the share space of proposed diagnosis by an iterative practice of searching-sharing-evaluation-used-searching until the needed information is located. 
Retrieval diagnosis: Students must decide which diagnosis they are likely to obtain to develop and evaluate different diagnoses by increasing the likelihood of a relevant diagnosis. Formulate alternative diagnoses, if necessary, or use the relevant shared probable diagnosis. The tutor encourages students to use only the most relevant probable diagnosis. The diagnosis used will appear in the students' used diagnosis space.

Finale diagnosis: The tutor's feedback is the key reference for confirming the students' final diagnosis. The collective diagnosis obtained is also compared and reevaluated collaboratively for common understanding and use. In the proposed model, iterative clinical actions are required to explore the information required before a final diagnosis can be used. Students use the clues obtained from the collective diagnosis to synthesize and decide on their final diagnosis.

Final treatment plan: Students should be encouraged to enhance their experience and expertise in designing treatment plans. Students develop a definitive treatment plan after a much more detailed explanation of the diagnosis. They can use the results of the final diagnosis from the previous steps to decide on their final treatment plan. Information needs are met when the synthesized information is used to solve the problem within the framework of clinical reasoning. The iterative process can be repeated until the students arrive at a relevant use of the information to solve the problem.

At the end, a summary form of this iterative process (meeting report) is obtained, which provides a simple and readable structure that summarizes the essence of the collaborative approach. Moreover, it provides an ideal reference for the reorganization and activation of knowledge. This step is important to allow knowledge transfer and application to other similar clinical situations. All the activities and reasoning actions performed by the students are actually stored in an assessment group, so that the tutor can evaluate the student's performance.

\section{Embodying Proposed Model Through a Collaborative Environment MEDcollab}

To assess performance, the proposed model needs to be refined and tested in real life. Thus, it was embodied in a collaborative environment (Web 2.0-based environment). This technology was chosen to benefit from its ease of use and technical performance. The second generation of Web tools is of particular importance in the field of education, as it constitutes a new and innovative pedagogical tool. It offers students and tutors opportunities for reflection; they collaborate and learn together, develop and share content using various tools and resources, and reuse and organize content according to their preferences and needs [27]. The best attributes of Web 2.0 are its tools for interaction, collaboration and can also enhance e-learning [28].

The MEDcollab platform offers students a better and more flexible opportunity to develop their behavioral skills in clinical reasoning in a framework specifically designed for their level. The implementation of a new pedagogical approach that assesses clinical reasoning can be adapted to all health care settings (cardiology, pediatrics, 
gynecology, etc.) and reach all levels of medical students. They are trained to identify, synthesize and perform a more in-depth assessment of a patient's condition before intervening.

The MEDcollab platform was implemented as a JAVA web application (JSP/ Servlet), running on the Apache Tomcat server, a combination that runs over the Internet or local area networks and supports any number of simultaneous CCRL2.0 meetings. The frontend (or also called GUI for Graphical User Interface) was realized with Bootstrap a jQuery. Bootstrap ensures a straightforward use interface; it makes web development faster and easier. It contains design templates based on HTML and CSS for responsive front-end design, as well as optional JavaScript plugins. jQuery ensures responsiveness and communication with the backend. It is a collection of widgets and GUI themes that is implemented with a JavaScript library. It is used to design websites in the backend. The backend was realized with Java and JSP, Java for Servlets, which communicate with the MySQL database, process frontend queries, etc., and the JSPs that are the basis of the communication between the frontend and the backend.

The subject of the meeting can be any pathology (pediatrics, cardiology, urology, gynecology, trauma). The clinicians (students and tutors) of a MEDcollab group can be in remote locations and can share the same environment within the network. There is no technical limit to the number of students in a MEDcollab group, but for practical and pedagogical reasons, a group generally includes no more than eight (08) students [7]. A chat feature for better communication and a notification system for important feedback was also implemented.

Although there are several tools for CR teaching and learning, to date there is no web 2.0 platform that fully supports collaborative behavioural reasoning skills in medical education. Thus, this work aims to propose a model dedicated to the design of a collaborative clinical reasoning behavioural environment in synchronous mode. The proposed model was concretized by a collaborative environment based on Web 2.0 technologies supporting collaborative clinical behavioural reasoning skills called MEDcollab.

The proposed environment is mainly based on the reasoning approach of the dual process theory, where the clinician formulates a concept using a non-analytical technique and then evaluates it using an analytical technique [29]. The MEDcollab platform has been built to meet the needs of this theoretical approach by providing interfaces for the tutor and students to acquire behavioural clinical reasoning skills.

Therefore, it seems significant to propose certain concepts (semantic representation, hypotheses, diagnosis, treatment plan) using a non-analytical technique and then to evaluate them collaboratively using an analytical technique because this proposal serves to optimize, in the student's memory, the identification of relevant diagnoses. The proposed model provides digital services for medical education to students and tutors. It is an e-learning platform, which creates an online collaborative learning environment based on Web 2.0 technologies, promoting exchanges and interactions between medical students and tutors in a medical educational content for the remote CCRL 2.0 meeting. The MEDcollab platform provides interfaces to students and tutors with numerous functionalities. 


\subsection{Tutor's interface}

In the first setting, the platform is private and accessible only to the tutor, who can manage the CCRL2.0 meeting and develop the clinical case data. The patient's task is delegated to the tutor. Tutors are the main actors who build and create CCRL2.0 sessions. The MEDcollab website requires tutors to register, create and configure the meetings. Each session contains only one specific clinical case and each tutor provides their own clinical cases (Figure 2).

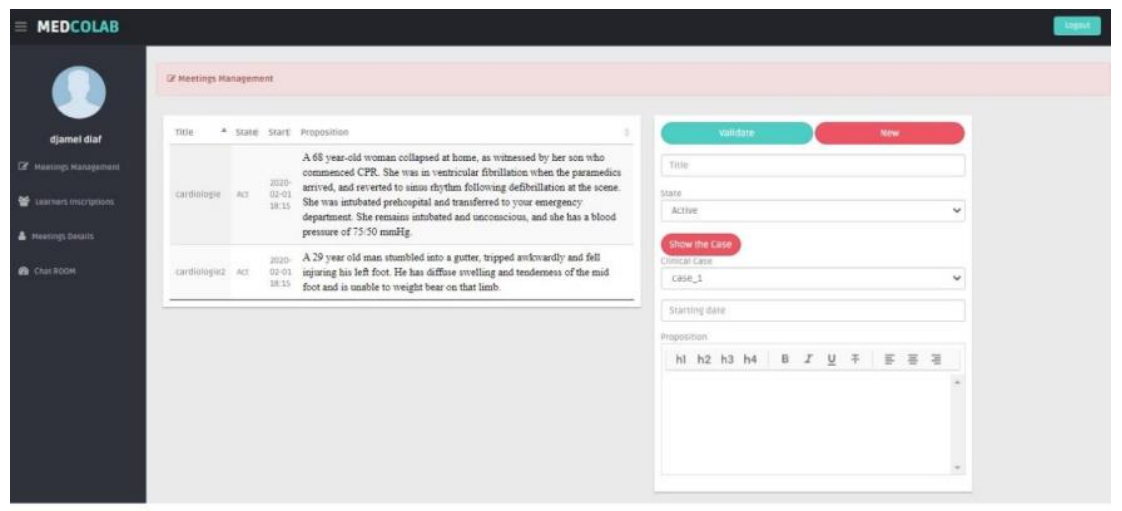

Fig. 2. Screenshot of CCRL2.0 Meeting management interface

MEDcollab supports an unlimited number of clinical cases. Cases consist of a summary of symptoms, physical exams, MRI (magnetic resonance imaging), CT images and laboratory tests, and electrocardiogram (ECG) images. After having developed meeting sessions, trainees are invited to register for the different sessions proposed by the tutors, so that the clinical tutor recommends the most appropriate case for each of them according to the registration information (profile).

The tutor can access all the details of the phases of the CCRL2.0 meeting. The session is a collaborative and interactive interaction, supervised by a tutor who manages the session and the time.

It allows the tutor:

a) To give feedback to the group or to an individual student requires a focus not only on clinical knowledge and decisions, but also on the resolution and analysis of clinical cases (Figure 3)

b) To comment on the results of the resolution, encourage students to focus their attention exclusively on the most important results and help them to correct their mistakes (Figure 3)

c) To follow students when evaluating resolution behaviour; for each suggestion included in the collection of tables, the tutor can consult more details (5 tables) providing more complete information on the selected proposal (Figure 3) 
d) To validate the transfer between phase; at the end of each phase, the tutor commits to validate only the most relevant hypotheses to improve its problem-solving process to allow the passage between the different phases of resolution (Figure 3)

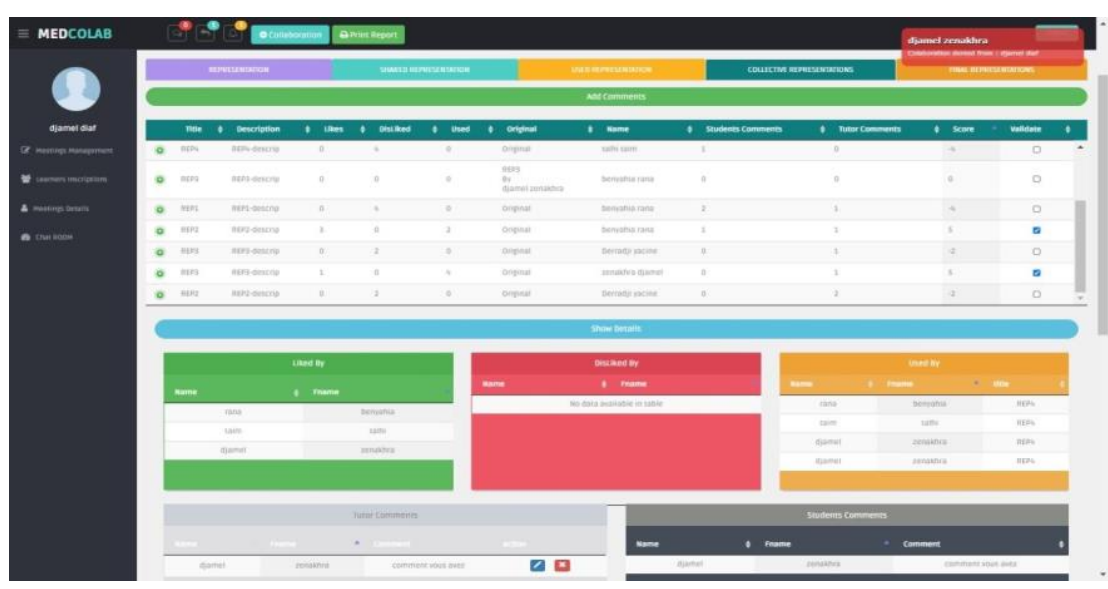

Fig. 3. Screenshot of CCRL2.0 meeting phases interface

e) To send a collaboration request; throughout a session, the tutor can ask students to collaborate by selecting the student's name from the list; the system sends a request message to the recipient; the program submits another message to the tutor who requested collaboration to inform him/her of the acceptance (Figure 4)
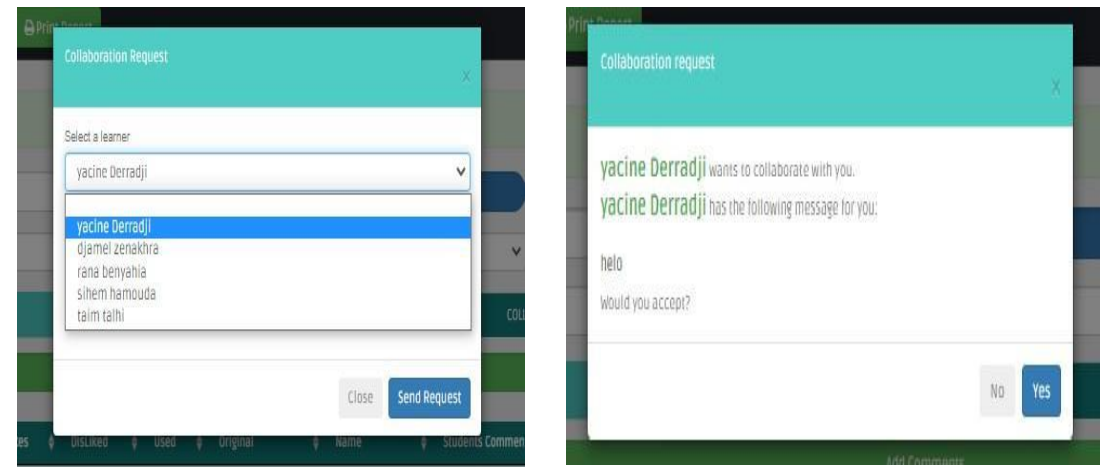

Fig. 4. Screenshot of Collaboration request

f) To evaluate group performance; at the end of the session, the tutor also has access to the report of the meeting; to help him/her evaluate the performance of the group participants, the resolution process considers potential errors and evaluates the group's participation (Figure 5). 


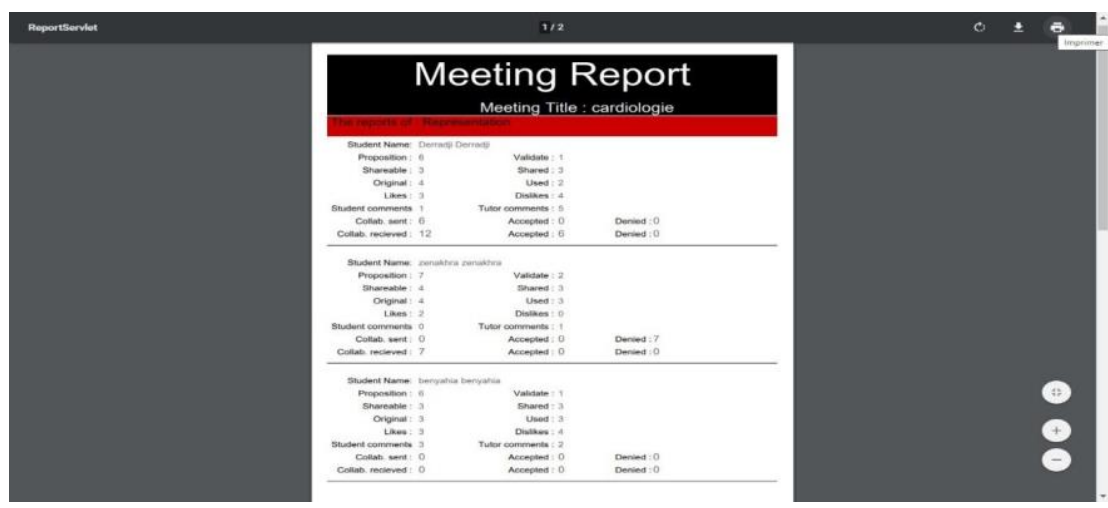

Fig. 5. Screenshot of meeting report

\subsection{Student's interfaces}

In the proposed environment, thanks to the integrated necessary means, the students can:

Refer to the clinical cases proposed in the clinical cases component: Students should be treated as a spontaneous report from a patient; this provides sufficient detail to encourage students to engage and make a relevant diagnosis (name, age, gender, patient history, physical examination, etc.) (Figure 6)

a) Submit new ideas: Share them with the group of students and update them from personal proposition editing (Figure 6)

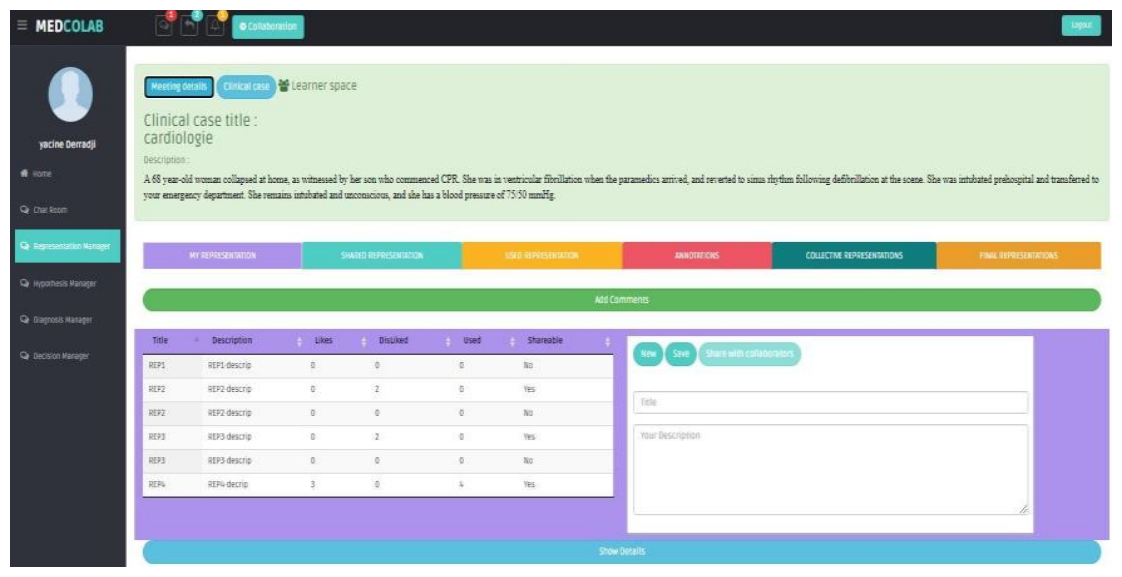

Fig. 6. Screenshot of private student interface

b) Collaboratively evaluate the shared proposal: Both the students and the tutor can access the shared results at any time to search for a proposal, submit remarks, 
questions and justifications by adding comment, use it to achieve personal goals and evaluate it with "Liked" or "Disliked" (Figure 7)

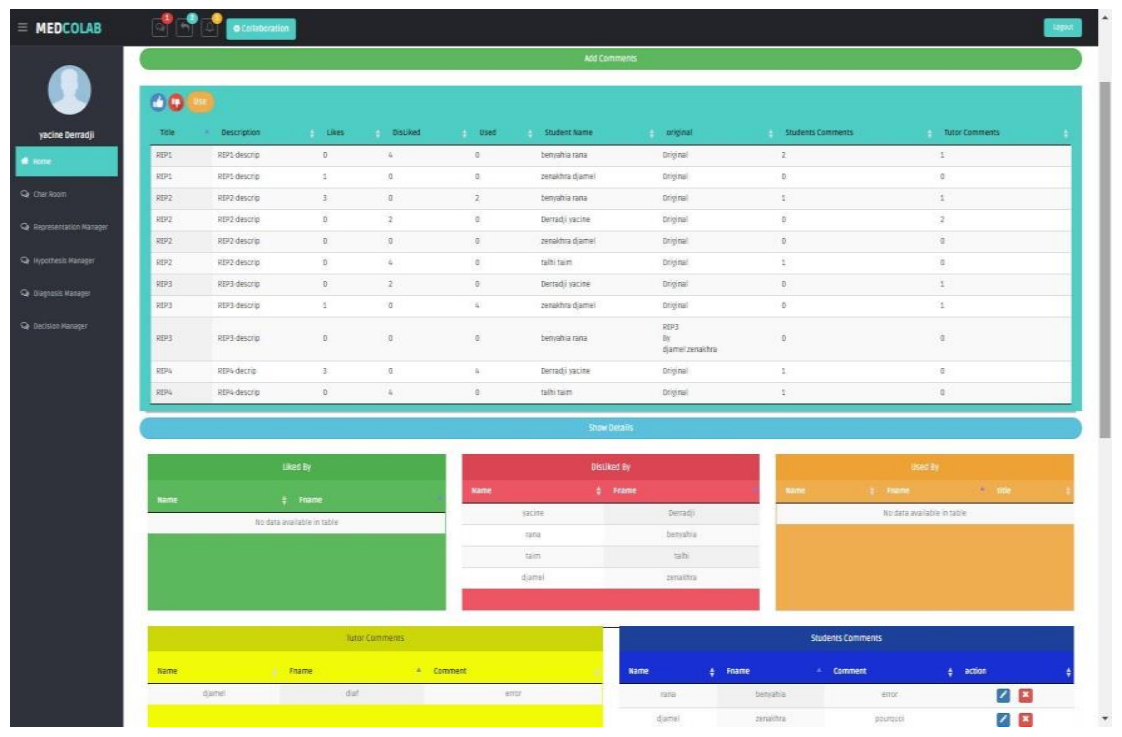

Fig. 7. Screenshot of collaborative interface

c) Re-evaluate the proposals based on annotation: Students may ask the tutor for additional details and additional tests necessary to evaluate and/or confirm the hypothesis. All students will share and show medical images relevant to the group. All students will see the same image and see what others are doing in the image (drawing, annotation and commentary, etc.). When a student points to and interacts on an area of the image, the image will be stored so that it can be seen by all students in the group. Each marked area is defined with the student's name and the correct one (Figure 8).

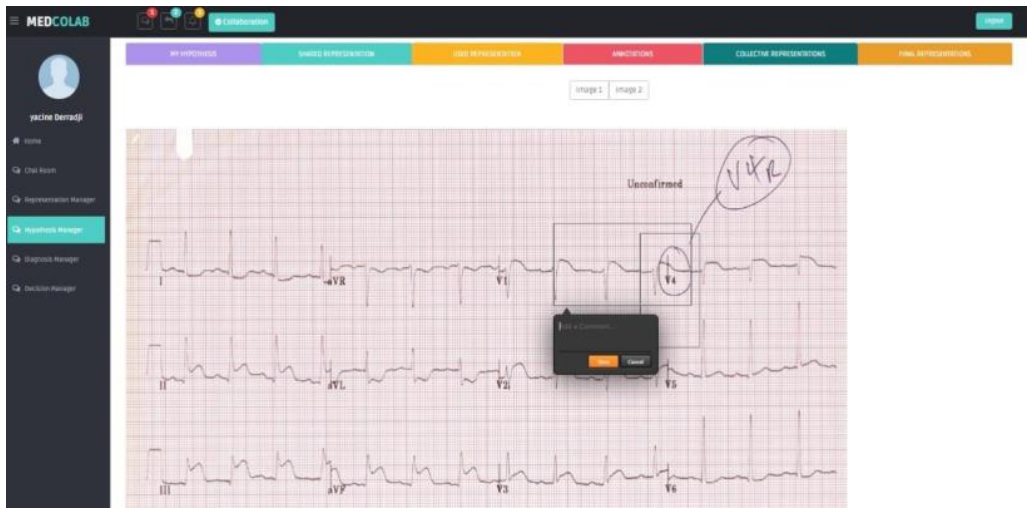

Fig. 8. Screenshot of annotation of supplementary test interface 
According to the annotation, the tutor encourages students to collectively and iteratively re-evaluate the results of each process. Each student must add comments, justifications or questions to re-evaluate the proposals. The tutor should participate and validate only the most important concepts to improve the problem-solving process.

\section{Experimentation: Methods}

CCRL0.2 sessions aim to help students acquire or apply knowledge, and to teach students a way of thinking like a physician.

\subsection{Participants and educational context}

This experiment was carried out during eight days in March 2020 at the University Badji Mokhtar and the Hospital Ibn-Rochd, in Annaba (northeastern Algeria). The CCRL 0.2 sessions were provided to external medical students. The students are in the sixth year of medicine. The research involved two professional clinicians (residents) to supervise the student groups. Two clinical cases were used as part of the research experience. They were selected by the clinical tutors involved. The case category was identified according to the level of the external students.

The first case was achalasia (gastroenterology) which is a rare disease, affecting 1 in 100,000 people, characterized by a loss of nerve cells in the esophageal wall. Achalasia can be diagnosed by X-ray, endoscopy or esophageal manometer. And the second case was represented by chest pain (cardiology) which is one of the most common symptoms that bring an individual to the emergency room. Seeking immediate care can save lives, and considerable public education has been undertaken to encourage patients to seek medical care when chest pain occurs. Some diagnoses of chest pain are life-threatening, while others are less dangerous [30].

These two clinical cases were chosen due to the pedagogical importance of medical students. The first case, achalasia, represents a rare situation and this allows students to practice rare cases. As for the second case, there are many causes of chest pain that a physician can think of. Some diagnoses are life-threatening, while others are less serious; this allows students to improve their ability to make a more detailed assessment of the medical situation before undertaking further interventions.

\subsection{Study design}

The study was carried out in three phases. The first phase represents the introductory phase: Training and preparation of the clinicians teaching the traineeship students and the CHU. All participants were initiated and sensitized to the session of the CCRL02 phase, one week before the launch of the demonstration of the real case using the MEDcollab platform. This was a 30-minute program during which both participants received an interactive video demonstrating the functionalities of the MEDcollab interface methodology, and had the opportunity to ask questions and explain their concerns. 
The second phase represents the sessions of CCRL 0.2 were developed and modelled. The CCRL0.2 sessions were developed by the clinicians' tutors. A registration is required in the MEDcollab platform for which the sessions would be designed and customized. Each session includes a single clinical case. Each tutor highlights his or her own clinical cases. The participating clinical tutors are the ones who choose the most appropriate clinical cases for the students. It was important to propose the cases according to the level of knowledge of the students. Groups of participating students were invited to register on the platform to allow clinical tutors to choose the most relevant cases according to their expertise.

And finally, the third phase is dedicated to the CCRL0.2 simulations which includes two studies. The first study was the feasibility of CCLR's 0.2 sessions. For this study, two types of CRL sessions were developed and the tutors were trained accordingly: a first type (group B) which did not work with the proposed method (classical CRL approach), and a second type (group A) which worked with the proposed approach of CCRL 0.2 session. The objective of this study is to evaluate the feasibility of the CCRL0.2 sessions to help students acquire or apply knowledge, to teach students a way of thinking in CRL, and to answer relevant questions in medical education:

- How to motivate the student to make a more detailed analysis of the medical situation before acting?

- How to promote learning and evaluation of abstract concepts such as clinical reasoning, negotiation, critical thinking skills?

The feasibility of the CCRL0.2 sessions was assessed by analysing the different phases to summarize what is expected from the students of the CCRL0.2 sessions by stimulating them to make a more detailed analysis of the medical situation before taking any action, and to summarize the results regarding student participation in the diagnosis. Ten students were eligible for this study: five in group B and five in the intervention group.

The second study aimed to assess the MEDcollab platform's usability. A seven-point questionnaire (Table 2) was used to test the usability of the MEDcollab platform, focusing on the choice of questions on the system's usability scale [31]. After each CCRL0.2 sessions, the online questionnaire became available. All participating students were invited to complete an online questionnaire via their email address.

\subsection{Learning outcome assessment}

At the end of the CCRL0.2 sessions, the evaluation of the results was assessed by examining specific dependent variable. According to CCRL0.2 sessions and other publications available in the literature [10] [11], the objective of preparing medical students to interpret clinical cases, to regularly perform each phase of clinical reasoning and to verbalize their thought processes with ease and without limits is to ensure that they are able to: 
- Represent a clinical case: suggest one or more representation, hypotheses, diagnosis and treatments plan

- Share one or more assumption

- Use one or more assumptions;

- Clinical data-gathering: identify the question and intervention

- Work together to identify an information need

- Accept feedback from other students or tutor

- Evaluate results

- Activate, develop and organize previous knowledge.

The primary outcome was to use the results (dependent variables) provided per group reports, which included: Number of proposed (representation, hypotheses, diagnosis, treatments plan); Number of representation validated by tutor for sharing; Number of shared (representations, hypotheses, diagnosis, treatments plan); Number of Evaluated (Number comparing comments, Number justifications); Number of Used (representations, hypotheses, diagnosis, treatments plan); Number of information seeking intervention; Number of justification intervention; Supplementary tests (Number of annotations, Number of questions oriented by annotations); Number of tutor feedbacks; Number of request collaboration sent to students and tutor (Accepted, Denied).

The students' experience was measured by taking their feedback on a 5-point Likert scale ranging from 'Disagree Strongly', 'Disagree Slightly', 'Agree Slightly', 'Agree Moderately' and 'Agree Strongly'.

\section{$5 \quad$ Results}

In Group B, the tutor notes the student's results. The tutor will also have access to a meeting report in the MEDcollab group to analyse the progress of the group participants, the interaction process, to recognize possible errors and to analyse the group's performance. Table 1 summarizes the outcomes of each dependent variable with all case experiences according to the groups of results. The data were analysed using IBM SPSS statistics 23 software.

Table 1. Results of each dependent variable with all case experiences according to the outcome groups

\begin{tabular}{|l|c|c|c|c|c|c|c|}
\hline & \multicolumn{9}{c|}{$\begin{array}{c}\text { MEDcollab group } \\
\text { N=5 }\end{array}$} & \multicolumn{3}{c|}{$\begin{array}{c}\text { Control group } \\
\text { N=5 }\end{array}$} \\
\hline \multicolumn{1}{|c|}{ Dependent variables } & Result & Mean & SD & Result & Mean & SD \\
\hline \multicolumn{1}{|c|}{ Suggest one or more REPRESENTATION } \\
\hline Nbr proposed representation & 45 & 9,00 & 0,71 & 37 & 7,40 & 5,32 \\
\hline Nbr validated representation by tutor for sharing & 35 & 7,00 & 0,71 & 20 & 4,00 & 2,24 \\
\hline Nbr shared representation & 33 & 6,60 & 0,55 & 19 & 3,80 & 1,30 \\
\hline $\begin{array}{l}\text { Evaluated representation (Nbr comparing comments, Nbr } \\
\text { justifications) }\end{array}$ & $69 ; 80$ & 39,00 & 2,55 & $26 ; 38$ & 11,80 & 3,49 \\
\hline Nbr used hypotheses & 30 & 6,00 & 0,71 & 15 & 3,00 & 1,22 \\
\hline Time of evaluated collective representation & $10 \mathrm{mn}$ & 2,00 & 0,00 & $5 \mathrm{mn}$ & 0,80 & 0,84 \\
\hline
\end{tabular}




\begin{tabular}{|c|c|c|c|c|c|c|}
\hline \multicolumn{7}{|c|}{ Suggest one or more HYPOTHESE } \\
\hline Nbr proposed hypotheses & 76 & 15,20 & 84 & 57 & 11,40 & 3,91 \\
\hline Nbr shared hypotheses & 73 & 14,60 & 1,34 & 35 & 7,00 & 2,00 \\
\hline $\begin{array}{l}\text { Nbr evaluated hypotheses (Nbr comparing comments, Nbr } \\
\text { justifications) }\end{array}$ & $\begin{array}{l}125 \\
210\end{array}$ & 62,40 & 2,70 & $56 ; 87$ & 13,60 & 3,29 \\
\hline Nbr used hypotheses & 100 & 11,60 & 0,55 & 20 & 4,00 & 1,58 \\
\hline Time of evaluated collective hypotheses & $15 \mathrm{mn}$ & 3,00 & 0,00 & $8 \mathrm{mn}$ & 1,40 & 1,67 \\
\hline \multicolumn{7}{|c|}{ Suggest one or more DIAGNOSIS } \\
\hline Nbr proposed diagnosis & 64 & 12,80 & 1,79 & 34 & 6,80 & 2,17 \\
\hline Nbr shared diagnosis & 56 & 11,20 & 0,84 & 12 & 2,40 & 1,34 \\
\hline $\begin{array}{l}\text { Nbr evaluated diagnosis (Nbr comparing comments, Nbr } \\
\text { justifications) }\end{array}$ & $89 ; 96$ & 46,80 & 2,49 & $45 ; 41$ & 22,40 & 13,45 \\
\hline Nbr used diagnosis & 76 & 15,20 & 0,45 & 24 & 4,80 & 2,28 \\
\hline Time of evaluated collective diagnosis & $15 \mathrm{mn}$ & 3,00 & 0,71 & $9 \mathrm{mn}$ & 1,40 & 1,67 \\
\hline \multicolumn{7}{|c|}{ Suggest one or more TREATMENT PLAN } \\
\hline Nbr proposed treatment plan & 15 & 3,00 & 0,71 & 5 & 1,00 & 1,22 \\
\hline Nbr shared treatment plan & 10 & 2,00 & 0,71 & 3 & 0,60 & 0,96 \\
\hline $\begin{array}{l}\text { Nbr evaluated treatment plan (Nbr comparing comments, } \\
\text { Nbr justifications) }\end{array}$ & $123 ; 80$ & 31,20 & 1,48 & $64 ; 44$ & 7,00 & 4,06 \\
\hline Nbr used treatment plan & 80 & 3,00 & 0,71 & 20 & 0,40 & 0,55 \\
\hline Time of evaluated collective treatment plan & $10 \mathrm{mn}$ & 2,00 & 0,00 & $5 \mathrm{mn}$ & 0,80 & 1,10 \\
\hline \multicolumn{7}{|c|}{ CLINICAL DATA-gathering } \\
\hline Nbr information seeking intervention & 129 & 25,80 & 1,79 & 85 & 11,20 & 2,59 \\
\hline Nbr justification intervention & 156 & 31,20 & 1,92 & 97 & 8,60 & 3,78 \\
\hline \multicolumn{7}{|c|}{ Annotations SUPPLEMENTARY TESTS } \\
\hline Nbr annotation & 69 & 13,80 & 3,70 & 38 & 6,80 & 4,76 \\
\hline Nbr questions oriented by annotations & 57 & 11,40 & 1,82 & 26 & 5,40 & 2,70 \\
\hline \multicolumn{7}{|c|}{ Tutor FEEDBACKS } \\
\hline Nbr feedbacks & 251 & 50,20 & 8,07 & 129 & 34,80 & 24,32 \\
\hline \multicolumn{7}{|c|}{ Request of COLLABORATION } \\
\hline Nbr Collab. Sent to learner & 82 & 16,40 & 0,55 & 43 & 7,40 & 4,72 \\
\hline Nbr Collab. Sent to tutor & 65 & 13,00 & 1,41 & 39 & 4,00 & 2,55 \\
\hline
\end{tabular}

\subsection{Problems' representations}

Proposed representation number: The student in the CCRL0.2 group (Group A) proposed more than students of the Group B (9 vs. 7.4, p value < 0.01$)$.

Validate Sharing Representation: Students using the MEDcollab platform were more motivated in their shared representation than students of the Group B (6.60 vs.3.80, $\mathrm{p}$ value $<0.01$ ).

Evaluated representation: Group A evaluated their probabilities of representation twice more than the Group B (39 vs.11.80, $\mathrm{p}$ value $<0.01$ ) by presenting supporting details from the clinical case, literature or background knowledge. During student interactions in the Group B, comparing and contrasting assessment possibilities seldom happened.

Used representation: Group A used relevant representation more than the Group B ( 6 vs. 3 , p value $<0.01$ ) to update and activate previous knowledge. 
Time of validate collective representation: The time taken for collaborating collective representation was greater in Group A as compared to Group B (10 mn vs. 5 $\mathrm{mn})$.

\subsection{Generating hypotheses}

Proposed hypotheses: Group A generated hypotheses more than twice as many as Group B hypotheses $(15.20$ vs. 11.40, p < 0.01$)$.

Shared hypotheses: Students using MEDcollab platform were more motivated in their shared hypotheses than Group B (14.60 vs. 7, p < 0.01).

Evaluated hypotheses: Group A evaluated their collective shared hypotheses twice more than the Group B (62.40 vs13.60, p < 0.01).

Time of validate collective hypotheses: The time taken for collaborating collective hypotheses was more twice in Group A as compared to Group B (15 min vs. $8 \mathrm{~min}$ ).

Used hypotheses: Group A used valid hypothesis revise and activate prior information more than the Group B (11.60 vs. 4 , p value < 0.01$)$.

\subsection{Diagnosis}

Proposed diagnosis: Students using MEDcollab platform identified more than double as many diagnoses as Group B (12.80 vs. 6.80, p value < 0.01$)$.

Shared diagnosis: Group A were more motivated in their shared representation than students of the Group B (11.20 vs. 2.40, p < 0.01).

Evaluated diagnosis: Group A assessed their diagnosis probabilities twice as high as the Group B (46.80 vs. $22.40, \mathrm{p}<0.01)$ by providing relevant details. Comparing and contrasting assessment possibilities seldom occurred during student experiences in the Group B.

Time of validate collective diagnosis: In MEDcollab platform, the time taken to validate collectives' diagnosis propositions was higher in comparison to the Group B (15 mn vs. $9 \mathrm{mn}$ ).

Used diagnosis: Group A used valid hypothesis revisit and trigger previous knowledge rather than the Group B (median 15.20 vs. 4.80, p value $<0.01$ ).

\subsection{Treatment plan}

Proposed Treatment plan: Group A proposed treatment plan more than students in the other group (3 vs. 1, p < 0.01 ).

Shared Treatment plan: In their shared treatment plan, students using MEDcollab platform were more inspired than Group B (2 compared to 0.6, p < 0.01).

Evaluated Treatment plan: Group A evaluated their probabilities of treatment plan twice more than the Group B $(31.20$ vs. 7 , p value < 0.01$)$ by presenting supporting details.

Used Treatment plan: Group A used relevant representation more than the Group B (median 3 vs. 0.40, p value < 0.01 ) to update and activate previous knowledge. 
Time needed to confirm collaborative results: Group A took on average $5 \mathrm{mn}$ more to validate their collective shared Treatment plan (10 mn vs. $5 \mathrm{mn})$.

\subsection{Clinical data-gathering}

Information seeking intervention to the story: Group A formulated nearly eight (08) times more questions than the students of Group B (25.80 versus 11.20, p value < $0.01)$.

Justification: Group A justified their diagnostic possibilities more than five (05) times more often than Group B $(31.20$ vs 6.80 , p value $<0.01)$.

\subsection{Annotations of supplementary tests}

Annotation: Group A justified their annotation more than twice times more often than Group B (13.80 vs 6.80, p value < 0.01).

Information seeking intervention oriented by annotations: In the CCRL 0.2 sessions, students (Group A) formulated almost twice as many questions as Group B (11.40 vs 5.40, p value $<0.01)$.

\subsection{Tutor feedbacks}

Tutor feedbacks of Group A is nearly threefold compared to Group B feedback $(50.2$ versus 34.80, $\mathrm{p}$ value $<0.01$ ).

\section{$5.8 \quad$ Request collaboration}

Request collaboration to student: Students using MEDcollab platform (Group A) were more motivated than students of the Group B. They accept to brainstorm and collaborate their shared ideas ( 16.40 vs 7.40 , p value $<0.01$ ).

Request collaboration to tutor: Students using the MEDcollab platform (Group A) were more motivated than students of the Group B. They accept to collaborate with tutor for filing the gaps of their clinical reasoning and to identify the need information (13 vs 4 , $\mathrm{p}$ value $<0.01)$.

\subsection{Usability questionnaire}

Table 2 highlights the feedback taken from students on five points Likert scale. Thirty (30) participants completed the questionnaire developed for this purpose. No usability problems were recorded. 
Table 2. Results of the usability questionnaire $(n=30)$, rated on a 5-point Likert scale $(1=$ Strongly disagree, $5=$ Strongly agree $)$.

\begin{tabular}{|l|c|c|c|c|}
\hline \multicolumn{1}{|c|}{ Questions } & Min & Max & Mean & SD \\
\hline I think that I would like to use MEDcollab platform frequently. & 1 & 5 & 3,67 & 1,061 \\
\hline I found MEDcollab platform unnecessarily complex. & 1 & 5 & 1,67 & 1,028 \\
\hline I thought MEDcollab platform was easy to use. & 1 & 5 & 4,30 & 1,119 \\
\hline I found the various functions well integrated in MEDcollab platform. & 1 & 5 & 3,93 & 1,230 \\
\hline I can explain my reasoning verbally at every step. & 2 & 5 & 4,27 &, 907 \\
\hline I can perfectly imitate the tutor's analytical abilities and behavior. & 2 & 5 & 4,23 &, 971 \\
\hline MED collab platform helps my reasoning organize. & 2 & 5 & 4,00 & 1,083 \\
\hline
\end{tabular}

The questionnaires showed that most students responded that they "Agree Moderately" or "Agree Slightly" with the frequent use and ease of use of MEDcollab (63\% for questions 1). For the frequent ease of use of MEDcollab most students responded that they "Agree Moderately" or "Agree strongly" (87\% for questions 3). Most students answered "Disagree Strongly" or "Disagree Slightly" on the unnecessary complexity of the MEDcollab platform (83\% for question 2). Student evaluations of the effectiveness of the CCRL0.2 sessions based on the last three questions of the study showed an increasing appreciation of how reasoning was organized and verbally clarified at each phase, mimicking the analytical skills of the professional clinician.

Moreover, approximately $(83 \%)$ of the students indicated that they agreed or strongly agreed that the performance of the CCRL0.2 allowed them to better understand how to explain their reasoning verbally without limitation, and $70 \%$ of the students indicated that they agreed or strongly agreed that they were more motivated and gained confidence by fully imitating the tutor's analytical skills (Figure 9).

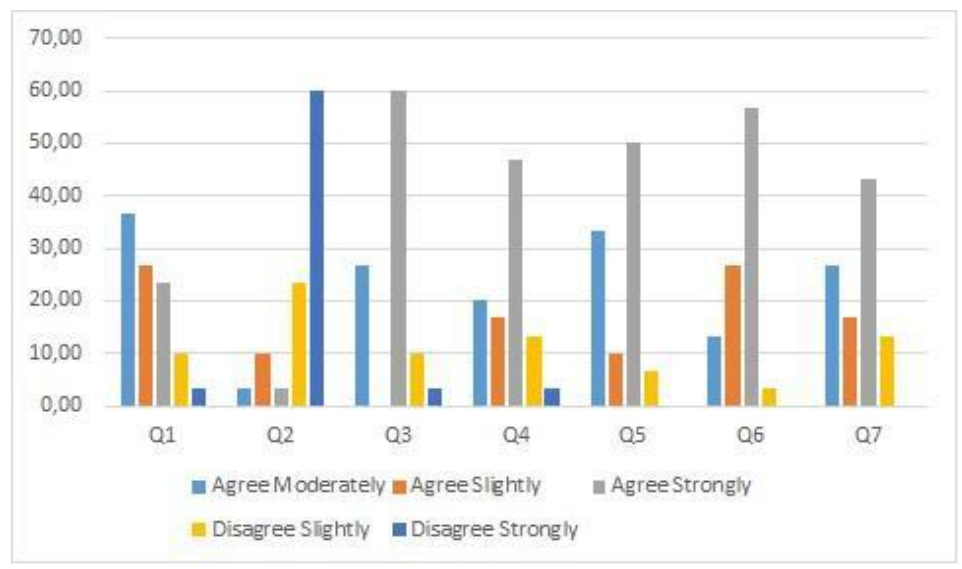

Fig. 9. Answers (\%) to feedback questionnaire. 


\section{Discussion}

The results of this research indicate that a collaborative learning environment is capable of increasing the perceived importance and value of medical education practices. This is significant for the future of translational medicine, as an appropriately skilled workforce is needed to improve medical therapies [32]. Previous systemic studies outside the medical field have shown that collaboration has significant beneficial effects on learning [21]. This study shows the feasibility of using collaboration and web 0.2 technology during CRL sessions for learning clinical reasoning and examining its effects on improving medical students' problem-solving and knowledge-building performance.

The study aimed to describe and explain how collaboration can motivate medical students to do a more detailed analysis of the medical situation before acting and how to promote the learning and evaluation of abstract concepts such as clinical reasoning and problem-solving skills. Results show that improving problem-solving skills and knowledge building performance has the greatest impact on the learning of clinical reasoning when using CCRL0.2 in the MEDcollab platform.

The CCRL0.2 student (Group A) participates actively in all phases of the session. While competent, the student responds in:

\subsection{Proposed assumption}

During CCRL0.2 sessions, the students (Group A) propose one more triple hypothesis (representation, hypotheses and diagnosis or treatment plan) than Group B. The ideal learning moment is when the student connects the new information to the prior knowledge network to display the information in an appropriate format [25]. Verbalization of clinical reasoning processes is a first step towards a better understanding of this skill [33].

\subsection{Shared one or more assumption}

Regarding the sharing of assumptions, CCRL0.2 (Group A) is more motivated than Group B. In medical education, students must be able to share their ideas confidently with other students, and must present themselves in a way that students can easily understand and use. The development of clinical reasoning (CR) evolves especially when clinicians share and interact on different points of view or even on a significant clinical experience [34].

\subsection{Evaluate results of each phase}

MEDcollab participants (Group A) reasoned aloud, discussing their analytical hypotheses more often than students in Group B. They analysed and evaluated their results, or justified any possible conclusions based on clinical findings. Throughout each problem-solving phase, students in Group B rarely explained their diagnosis. In our 
approach, the students use the "double process theory" where the hypothesis is proposed using a non-analytical strategy and then confirmed using an analytical strategy. According to Collard, this is one of the most effective professional pedagogical techniques for explaining the clinician's reasoning. Analytical approaches, such as comparing diagnostic possibilities, or justifying the diagnosis based on information retrieval, can provide evidence confirming a diagnostic possibility initially created by the student using a non-analytical technique. The most likely mechanism for describing the clinician's approach appears to be the use of a mixed process during the assessment of a clinical case [35]. Medical educators cannot assess the extent of the student's diagnostic reasoning without understanding the student's diagnostic hypotheses [36].

\subsection{Time needed to confirm collaborative results}

The MEDcollab students (Group A) initiated the collaboration of results, checking about $30 \%$ more often than Group B. This collaboration allows the tutor to appreciate the student's experience and to develop the evaluation program that follows. It is the ideal time to help the tutor understand the student's level to give immediate feedback and clarify any doubts [36]. Assessing the hypothesis is significant in the reasoning process that gives meaning to the information [37].

\subsection{Used one or more assumption}

Students in the control group used even fewer hypotheses than those in Group A. Students need to be aware and responsive, otherwise learning cannot take place [38]. Therefore, students must have the ability to apply the knowledge collected and use it successfully to achieve a particular goal. The use of relevant diagnostic hypotheses to define data collection appears to enhance the relevance of collected data and diagnostic skills [24].

\subsection{Information seeking intervention to the story}

The students in Group A provided the information they needed and about twice asked for help from the tutor or other students in the same group. Throughout the CCRL0.2 sessions, all the students in Group A expressed their difficulties almost every time. There was much less uncertainty about communication in Group B. Collaboratively seeking additional information is a crucial step in our approach. Students need to be able to fill in gaps in their clinical reasoning and identify the necessary information [1]. A request for additional data to validate or refute suggested conclusions. This data includes additional testing of the patient. The requested data will be sent by the tutor if it exists in the clinical case, and the tutor may ask the students to justify the need for the data. Collaborative annotation of these data and evaluation of hypotheses is done using the annotation tool. 


\subsection{Tutor's feedbacks}

CCRL0.2 session provided tutors with a student-initiated overview of the reasoning process and students' levels of understanding and uncertainty. As Khumrin reported, feedback to medical students is crucial to the successful development of their clinical reasoning skills [23]. For Voges and Frantz, clinical supervisors play an important role in helping students integrate theoretical and clinical knowledge [39]. If they make a mistake, feedback from tutors will direct specific information needs, recognize and report not only misdiagnoses but also inadequate detail, and enable students to improve their learning needs. Students can verify their clinical performance thanks to feedback from teachers [16].

\subsection{Request collaboration}

The reduced collaboration of Group B students made it difficult for the tutor to understand the student's clinical reasoning and also made it difficult to give effective feedback. It is useful to recognize the different levels of clinical reasoning when supervising a medical student to understand when the student finds it difficult to make the appropriate choice [40]. Cicourel highlighted the importance of the collaboration of actors in the diagnostic process [41]. One of the advantages of collaboration is that it allows participants to achieve more than they can achieve individually [18]. Students need to be able to understand teamwork and to brainstorm and collaborate by sharing ideas.

\subsection{Behavioural skills}

One significant result of this work is the increased confidence of the students in their unrestricted ability to conduct a clinical case. In the proposed collaborative environment, the student acquires clinical reasoning behavioural skills [42]:

- Seeking and searching skills: The ability to fill gaps in their clinical reasoning (CR) and identify the need for information.

- Presenting and sharing skills: The ability to present information in an appropriate format, share information confidently with other students, and present information in a way that can be easily understood and used.

- Used skills: The ability to organize the information collected according to their knowledge and expertise and to successfully use the information to achieve the objective.

- Evaluating skills: The ability to accept feedback from other students or the tutor and to evaluate one's own performance based on suggestions from examiners to activate and reorganize knowledge.

- Team working skills: The ability to accept teamwork and to collaborate with other students or tutors, the ability to reflect and collaborate on a shared idea (hypothesis) in a group. 


\section{Conclusion and Further Work}

Clinical Reasoning (CR) is difficult to teach and practice because it is complex, implicit and virtually unknown to students. Self-cause research can only promote the learning of complex concepts such as clinical reasoning (CR) by contributing to effective learning. Therefore, the main objective of this work was to create a computer-based tool for medical e-learning in a collaborative form.

This paper described the main characteristics of a collaborative environment that supports the behavioural competencies of collaborative clinical reasoning based on Web 2.0 technologies dedicated to online medical learning (MEDcollab). The results show the feasibility of integrating collaboration into CRL sessions. The CCRL0.2 sessions significantly encourages and promotes the development of clinical reasoning. It will significantly improve the student's ability to make a more detailed assessment of a medical situation before undertaking a subsequent intervention. CCRL0.2 requires students to conduct a clinical case using a technique that makes each process explicit and provides students with the opportunity to justify their clinical reasoning and complexity. Finally, it reinforces professionalism in behaviour.

However, the study has some limitations. First, preliminary results from a small number of participants may not be sufficient to affirm the effectiveness of the approach for a larger population. Second, the research was conducted in a single medical hospital. Adaptation of this research model to other settings will be evaluated in the future. Its potential application to other health care disciplines, such as dentistry and nursing, will also be explored.

\section{$8 \quad$ References}

[1] Min Simpkins, A. A., Koch, B., Spear-Ellinwood, K., \& St. John, P. (2019). A developmental assessment of clinical reasoning in preclinical medical education. Medical education online, 24(1), 1591257. https://doi.org/10.1080/10872981.2019.1591257

[2] Linsen, A., Elshout, G., Pols, D., Zwaan, L., \&Mamede, S. (2018). Education in clinical reasoning: an experimental study on strategies to foster novice medical students' engagement in learning activities. Health Professions Education, 4(2), 86-96. https://doi. org/10.1016/j.hpe.2017.03.003

[3] Delany, C., \& Golding, C. (2014). Teaching clinical reasoning by making thinking visible: an action research project with allied health clinical educators. BMC medicaleducation, 14(1), 20. https://doi.org/10.1186/1472-6920-14-20

[4] Higgs, J., and Jones, M. A. (2008). Clinical decision making and multiple problem spaces. In Clinical reasoning in the health professions (3rd ed. / 1 ed., pp. 3-18). Butterworth-Heinemann.

[5] Carr, S. (2004). A framework for understanding clinical reasoning in community nursing. Journal of Clinical Nursing, 13 (7), pp. 850-857. https://doi.org/10.1111/j.13652702.2004.00959.x

[6] Charlin, B., Lubarsky, S., Millette, B., Crevier, F., Audétat, M. C., Charbonneau, A., $\&$ Bourdy, C. (2012). Clinical reasoning processes: unravelling complexity through graphical representation. Medical education, 46(5), 454-463. https://doi.org/10.1111/j.1365$\underline{2923.2012 .04242 . x}$ 
[7] Ortega, E. M., Lessard, Y., Burgun, A. \&Le Beux, P. (2005). Virtu@1Consult@tion: an interactive and multimedia environment for remote clinical reasoning learning in cardiology. Computers in Cardiology, IEEE.C, September 2005. pp. 829-832. https://doi.org/10.1109/ cic. 2005.1588233

[8] Hege, I., Kononowicz, A. A., Kiesewetter, J., \& Foster-Johnson, L. (2018). Uncovering the relation between clinical reasoning and diagnostic accuracy-an analysis of learner's clinical reasoning processes in virtual patients. PloS one, 13(10), e0204900. https://doi.org/ 10.1371/journal.pone. 0204900

[9] Welch, P., Young, L., Johnson, P., \& Lindsay, D. (2018). Metacognitive awareness and the link with undergraduate examination performance and clinical reasoning. MedEdPublish, 7(2). https://doi.org/10.15694/mep.2018.0000100.1

[10] Gouzi, F., Hédon, C., Blervaque, L., Passerieux, E., Kuster, N., Pujol, T., Mercier, J., and Hayot, M. (2019). Interactive whiteboard uses in clinical reasoning sessions to teach diagnostic test ordering and interpretation to undergraduate medical students. BMC medical education, 19(1), 424. https://doi.org/10.1186/s12909-019-1834-1

[11] Nendaz, M. R., Gut, A. M., Louis-Simonet, M., Perrier, A., \& Vu, N. V. (2011). Bringing explicit insight into cognitive psychology features during clinical reasoning seminars: a prospective, controlled study. Education for Health, 24(1), 496.

[12] Iyer, S., Goss, E., Browder, C., Paccione, G., \&Arnsten, J. (2019). Development and evaluation of a clinical reasoning curriculum as part of an Internal Medicine Residency Program. Diagnosis, 6(2), 115-119. https://doi.org/10.1515/dx-2018-0093

[13] Dumas, J. P. (2017). L'évaluation du raisonnement clinique en physiothérapie. Thèse en sciences de l'éducation. Université de Montréal.

[14] Torre, D. M., Hernandez, C. A., Castiglioni, A., Durning, S. J., Daley, B. J., Hemmer, P. A., \&LaRochelle, J. (2019). The Clinical Reasoning Mapping Exercise (CResME): a new tool for exploring clinical reasoning. Perspectives on medicale ducation, 8(1), 47-51. https:// doi.org/10.1007/s40037-018-0493-y

[15] Lew, S. L., \& Lau, S. H. (2020). An Empirical Study of Students' Intention to Use Cloud ELearning in Higher Education. International Journal of Emerging Technologies in Learning (iJET), 15(09), 19-38. https://doi.org/10.3991/ijet.v15i09.11867

[16] Messineo, L., \& Allegra, M. (2014). An educational model for undergraduate psychiatry students to promote clinical diagnostic reasoning. Procedia-Social and Behavioral Sciences, 141, 1309-1314. https://doi.org/10.1016/j.sbspro.2014.05.224

[17] Iqbal, M., Velan, G. M., O'Sullivan, A. J., \&Balasooriya, C. (2016). Differential impact of student behaviours on group interaction and collaborative learning: medical students' and tutors' perspectives. BMC Medical Education, 16(1), 1-11. https://doi.org/10.1186/s12909016-0730-1

[18] Green, B. N., \& Johnson, C. D. (2015). Interprofessional collaboration in research, education, and clinical practice: working together for a better future. Journal of Chiropractic Education, 29(1), 1-10. https://doi.org/10.7899/jce-14-36

[19] Reddy, M.C. \&Jansen, B.J. (2008). A model for understanding collaborative information behavior in context: A study of two healthcare teams. Information Processing and Management, 44 (2008), pp. 256-273. https://doi.org/10.1016/j.ipm.2006.12.010

[20] Karunakaran, A., Reddy, M. C., \& Spence, P. R. (2013). Toward a model of collaborative information behavior in organizations. Journal of the American Society for Information Science and Technology, 64(12), 2437-2451. https://doi.org/10.1002/asi.22943

[21] Shah, C. \&Leeder., C (2015). Exploring collaborative work among graduate students through the C5 model of collaboration: A diary study. Journal of Information Science. https://doi.org/10.1177/0165551515603322

[22] Al Mulhim, E., \&Eldokhny, A. (2020). The Impact of Collaborative Group Size on Students' Achievement and Product Quality in Project-Based Learning Environments. International 
Journal of Emerging Technologies in Learning (iJET), 15(10), 157-174. https://doi.org/10. 3991/ijet.v15i10.12913

[23] Khumrin, P., Ryan, A., Juddy, T., \&Verspoor, K. (2018). DrKnow: A Diagnostic Learning Tool with Feedback from Automated Clinical Decision Support. In AMIA Annual Symposium Proceedings (Vol. 2018, p. 1348). American Medical Informatics Association.

[24] Audétat, M. C., Laurin, S., Dory, V., Charlin, B., \&Nendaz, M. R. (2017). Diagnosis and management of clinical reasoning difficulties: Part I. Clinical reasoning supervision and educational diagnosis. Medical Teacher, 39(8), 792-796. https://doi.org/10.1080/0142159x. 2017.1331033

[25] Nendaz, M., Charlin, B., Leblanc, V., \&Bordage, G. (2005). Le raisonnement clinique: données issues de la recherche et implications pour l'enseignement. Pédagogie médicale, 6(4), 235-254. https://doi.org/10.1051/pmed:2005028

[26] Qu, Y. \&Hansen, D.L. (2008). Building shared understanding in collaborative sense making. In Proceedings of the Workshop on Sense making at the Conference on Human Factors in Computing Systems (CHI). Florence, Italy

[27] Yadav, A., \&Patwardhan, A. (2016). Use and impact of web 2.0 tools in higher education: a literature review. Academic Libraries in Electronic Environment (pp.218-246). http://dx. doi.org/10.13140/RG.2.1.2748.6965/1

[28] Pontes, E., Kofuji, S., \&Guelfi, A. (Eds.). (2012). Methodologies, Tools and New Developments for E-Learning. BoD-Books on Demand. Croatia: InTech, ISBN 978-953-51-00294, 332 pages. https://doi.org/10.5772/1115

[29] Guyete, D., and Rinaudo, J-L. (2013). La formalisation et l'apprentissage du raisonnement clinique en formation initiale de masseur kinésithérapeute : une identification des savoirs de la pratique professionnelle. Département des Sciences de l'Education, Université de Rouen, France, 2013. https://doi.org/10.1016/s1779-0123(11)75175-0

[30] Moores, D. (2019). Healthline. Medically reviewed by Alana Biggers, MD_Updated on November 6, 2019. [Accessed 01-03-2020]. https://www.healthline.com/health/

[31] Usability. System Usability Scale (SUS). https://www.usability.gov/how-to-and-tools/methods/system-usability-scale.html. [accessed 30-03-2020]. https://doi.org/10.1201/97814987 $\underline{10411-35}$

[32] Makransky, G., Bonde, M. T., Wulff, J. S., Wandall, J., Hood, M., Creed, P. A., Bache, I., Silahtaroglu, A., \&Nørremølle, A. (2016). Simulation based virtual learning environment in medical genetics counseling: an example of bridging the gap between theory and practice in medical education. BMC medical education, 16(1), 1-9. https://doi.org/10.1186/s12909016-0620-6

[33] Faucher, C., Tardif, J., \&Chamberland, M. (2012). Optometrists' clinical reasoning made explicit: a qualitative study. Optometry and Vision Science, 89(12), 1774-1784. https://doi. org/10.1097/opx.0b013e3182776002

[34] Plante, H. \&Larue, C. (2014). The development of clinical reasoning for newly qualified nurses in critical care. Dissertation, Faculté des sciences infirmières, Université de Montréal, Canada, 2014.

[35] Collard, A., Bourguignon, J-P. \& Bredart, S. (2014). Le développement du raisonnement biomédical et clinique au cours du cursus médical. Dissertation, Belgique Université de Liège, 2014. https://orbi.uliege.be/bitstream/2268/182006/1/Th\%C3\%A8se\%20Anne\%20 Collard.pdf

[36] Jain, V., Rao, S., \&Jinadani, M. (2019). Effectiveness of SNAPPS for improving clinical reasoning in postgraduates: randomized controlled trial. BMC medical education, 19(1), 224. https://doi.org/10.1186/s12909-019-1670-3

[37] Chamberland, M. (2007). Les séances d'apprentissage du raisonnement clinique (ARC) description de la méthode pédagogique. Université de SHERBROOKE.

[38] Kurt, S. (2019). Using Bloom's Taxonomy to Write Effective Learning Objectives: The ABCD Approach. Educational Technology, April 24, 2019. https://educationaltechnology. 
net/using-blooms-taxonomy-to-write-effective-learning-objectives-the-abcd-approach/. https://doi.org/10.1016/b978-0-12-814702-3.00025-1

[39] Voges, T.-L. \&Frantz, J.M. (2019). Clarifying the role of clinical supervisors according to physiotherapists at a higher education institution', South African Journal of Physiotherapy 75(1), a523. https://doi.org/10.1097/ACM.0b013e3181d5dd0d. https://doi.org/10.4102/ sajp.v75i1.523

[40] Audétat, M. C., Rieder, A., \&Sommer, J. (2017). Teaching clinical reasoning is more like detective work than you might imagine. Revue medicale suisse, 13(562), 981-985.

[41] Cicourel, A.V. (2002). Medical Reasoning. A socio cognitive approach. Seuil, Paris, 2002.

[42] Santharooban, S. \&Premadasa, P. G. (2015). Development of an information literacy model for problem-based learning. Annals of Library and Information Studies, Vol. 62, September 2015, pp.138-144.

\section{Authors}

Gherib teldja is a PhD student in computer science at Badji-Mokhtar Annaba University (P.O. Box 12, 23000, Annaba, Algeria). Member of the E-Learning Research Group (GReLearn) of the Laboratory of Research in Computer Science (LRI). Her research areas include e-learning and training, educational computing, Human-machine Interaction Technology, and e-health. Email: teldja.gherib@univ-annaba.org

Tahar Bouhadada is a Professor at Badji-Mokhtar Annaba University (P.O. Box 12, 23000, Annaba, Algeria), Head of the E-Learning Research Group (GReLearn) and Head of Research Projects. Previously, Head of the Computer Science Department; Head of the pedagogy at the Computer science department, Head of the Laboratory of Research on Computer Science (LRI. His research areas include information systems, databases, distance learning environments, pedagogical agents, e-learning, social network exploration, Human-machine Interaction Technology, e-health and computer science in medicine. Email: bouhadada.tahar@univ-annaba.org

Article submitted 2020-10-27. Resubmitted 2020-11-30. Final acceptance 2020-12-01. Final version published as submitted by the authors. 